\title{
A note on the parallel processing of single-featured forms
}

\author{
STUART M. KEELEY ${ }^{1}$ AND MICHAEL E. DOHERTY \\ BOWLING GREEN STATE UNIVERSITY
}

\begin{abstract}
Single-featured forms (vertical and horizontal lines) were presented to Ss both successively and simultaneously, the same form being presented one, two, or four times. Hit rates (HRs) for both simultaneous and successive presentations of two and four forms exceed the single-form $H R$. The $H R$ for four successively presented forms (1F4) exceeds the four simultaneous form (4F) HR. The HR increases in the simultaneous conditions were not found in a previous study using single-featured forms. The discrepant results of the two studies indicate that energy level of stimulus presentation and confusability among forms affect the magnitude of $H R$ increase in the simultaneous conditions. The finding of a greater IF4 HR than 4F agreed with previous findings, indicating that with single-featured stimulus forms, $S$ s do not process simultaneous displays in parallel.
\end{abstract}

A recent study investigating the processing of simultaneously presented visual stimulus forms provided contradictory results, dependent upon the nature of the forms used (Keeley \& Doherty, 1968). With multidimensional forms (the graphemes A, T, or U), empirical hit rates (HRs) for multiple redundant form stimuli exceeded HRs for single-form stimuli. However, with singlefeatured forms, Landolt rings (Cs), Ss were no more accurate when four $\mathrm{Cs}$ were presented simultaneously than when a single $\mathrm{C}$ was presented. In addition, HRs for four successive presentations (1C4) of the same form significantly exceeded the HR for four simultaneous presentations $(4 \mathrm{C})$ of the form. The above evidence indicates that Ss' performance in the $4 \mathrm{C}$ condition cannot be represented by any model assuming multiple independent opportunities to perceive (i.e., parallel processing).

An essential difference between the $\mathrm{Cs}$ and the graphemes is the number of features discriminating the individual forms. The authors suggested that the lack of $\mathrm{HR}$ increase in the $4 \mathrm{C}$ condition may have reflected the fact that only one feature was available for analysis, irrespective of the number of forms. If an error were made in analyzing that feature, no correction would be possible in terms of detecting other features, as might be the case with multifeatured forms.

Also, in Experiment 1 of Keeley and Doherty (1968), Ss frequently made "confusion" errors, or the perceiving of the critical feature clearly, but erroneously. For example, during multiple-form presentations, Ss often reported seeing gaps in multiple directions even when they knew that all gaps were in the same direction. In addition, a fairly large number of trials on which "certain" confidence judgments were made were in error.

In order to test the generality of our earlier results with other single-featured stimulus forms and to reexamine the importance of confusion errors to our findings, we essentially replicated the previous study, changing the single-featured forms from $\mathrm{Cs}$ to straight lines. This change greatly reduced the exposure duration required to detect a single form at a level of accuracy greater than chance but low enough to permit an increase in HR in the multiple conditions. Also, with these stimuli, confusion errors were virtually eliminated. Thus, a comparison of these results with previous findings was expected to help clarify the effect of confusion errors on empirical HRs for multiple-form presentations.

\section{Subjects \\ Three graduate assistants who served in the previous investigation participated in this study.}

\section{Apparatus}

A Scientific Prototype Model GB tachistoscope with a handswitch permitting Ss to initiate stimulus onset was used.

\section{Stimuli}

The stimulus forms were vertical and horizontal lines. The lines were located at the 45-, 135-, 225-, and 315-deg positions on an imaginary circle (considering the top to be $0 \mathrm{deg}$ ) centered on a fixation point. The circle subtended a visual angle of $1.8 \mathrm{deg}$, the length of the line $.25 \mathrm{deg}$, the width of the line $.07 \mathrm{deg}$. The minimum separation between forms was $1.2 \mathrm{deg}$.

A single form stimulus (IF) had a line, either vertical or horizontal, at one of the four loci. Multiple form stimuli contained either two forms at diametrically opposite loci (2F) or four forms $(4 \mathrm{~F})$. The stimuli were transilluminated, and were mounted in square slides. Only one slide, therefore, was required for each stimulus display size.

\section{Procedure}

Viewing was monocular, with the preferred ofe Si rude forced-choice responses of "vertical" or "horizont 1 " plus confidence judgments indicating whether $S$ "felt sure," "thw wht so," "was guessing," or "saw nothing "These wer din biced ty $\mathrm{S}$ as "1," "2," "3," or "4," respectively. Stimulus presi lation was always initiated by $S$ after a ready signal, the sound if the stimulus being inserted.

Pretraining consisted of one $1 \mathrm{~F}$ stimulus per trial and continued until an exposure duration was found that yielded a stable IF HR between .70 and .80 . This duration was then used for a given $S$ across all subsequent conditions. Fxposure duratıons were $1.3,1.3$, and $1.5 \mathrm{msec}$.

Each experimental session included 8 practice and 60 test stimulus presentations. For simultaneous presentation sessions, $\mathrm{Ss}$ were randomly presented $\mathrm{IF}, 2 \mathrm{~F}$, and $4 \mathrm{~F}$ stimuli, with the restriction placed upon the randomization that 20 test trials of each be presented within a session. In the successive condition sessions there were two blocks of 10 trials of each condition. In one block two consecutive IF stimuli were presented before a response, with the direction of the line the same but the loci randomized. This defines the $1 \mathrm{~F} 2$ condition. The $1 \mathrm{~F} 4$ condition was defined similarly, with four stimuli before a response. The order of the blocks was counterbalanced across sessions. One simultaneous presentation session was always followed by two successive presentation sessions.

Subjects participated in 17 experimental sessions, each session taking approximately $50 \mathrm{~min}$. There were five simultaneous presentation sessions (100 trials each of $1 \mathrm{~F}, 2 \mathrm{~F}$, and $4 \mathrm{~F}$ presentations) and 12 successive presentation sessions (120 trials each of $1 \mathrm{~F} 2$ and $1 \mathrm{~F} 4$ presentations). Within a simultaneous session, line direction was randomized with the restriction that an equal number of vertical and horizontal stimuli occurred. Within 
Table 1

Obtained HRs for Each $S$ for Each of the Five Experimental Conditions

\begin{tabular}{lccccc}
\hline $\mathrm{S}$ & $1 \mathrm{~F}$ & $2 \mathrm{~F}$ & $4 \mathrm{~F}$ & $1 \mathrm{~F} 2$ & $1 \mathrm{~F} 4$ \\
1 & .74 & .84 & .83 & .79 & .92 \\
2 & .76 & .86 & .86 & .79 & .95 \\
3 & .76 & .81 & .91 & .83 & .93 \\
\hline
\end{tabular}

a successive session, each 1F2 and 1F4 block contained an equal number of vertical and horizontal presentations. Since stimulus changing was manual, the interpresentation interval was several seconds.

An experimental session began with about $10 \mathrm{~min}$ of dark adaptation followed by eight practice stimuli. The $\mathrm{S}$ was instructed to initiate presentation when fixation point was clearly focused.

\section{RESULTS}

Table 1 presents the obtained HR values for each $S$. The $2 F$ $\mathrm{HR}$ exceeds the $1 \mathrm{~F}$ for all three $\mathrm{Ss}$, but the $4 \mathrm{~F}$ exceeds the $2 \mathrm{~F}$ for only a single $S$. Thus, the most marked $H R$ increase in the simultaneous condition occurs as the number of forms increases from one to two. Both the $2 \mathrm{~F}$ and $4 \mathrm{~F}$ HRs exceed the $1 F \mathrm{FR}$, which contrasts with our previous finding of no increase in HR when $4 \mathrm{Cs}$ were simultaneously presented over a single $\mathrm{C}$ presentation. In the successive condition, HRs increase for all Ss as the number of forms presented increase. Also, the 1F4 HR exceeds the 4F HR for all Ss, which is consistent with our previous finding that the successive four form HRs exceeded the simultaneous four form HRs. No consistent differences are found between the $1 \mathrm{~F} 2$ and $2 \mathrm{~F}$ HRs.

\section{DISCUSSION}

A most interesting finding is the increase in $\mathrm{HR}$ in the $2 \mathrm{~F}$ and $4 \mathrm{~F}$ conditions over the $1 \mathrm{~F} \mathrm{HR}$. This increase was not found when Landolt $\mathrm{Cs}$ were the stimulus forms. Both Cs and straight lines can be considered single-featured forms. The question thus arises as to what accounts for the difference in results.

There are two basic differences between this study and the previous one. One is the lower energy level used, leading to many trials on which S "saw nothing." The other is the lack of confusion errors in this investigation. Under these conditions, increasing the number of stimulus forms does increase the HR, at least through two forms. Evidence for a reduction of confusion errors comes from subjective reports of the Ss and also their use of confidence judgments. In the present investigation Ss did not report seeing lines in different directions during multiple form presentations. Most errors appeared to be due to a lack of information. The subjective reports are supported by confidence judgment data. The mean error rates for " 1 " and " 2 " confidence judgments for the $4 \mathrm{~F}$ condition was $3 \%$ for the " 1 " judgments and $15 \%$ for the " 2 " judgments. This contrasts to error rates of 23 and $50 \%$ for the $4 \mathrm{C}$ condition in the previous study, which are markedly worse than in the present study even when the differential chance HRs are taken into account. It is apparent then that Ss" criterion for " 1 " and " 2 " judgments differed in the two studies; and one possible explanation is the perception of fairly clear, but erroneous information in the former study (errors that we have called confusion errors) and the absence of such errors in the latter study.

It is also interesting to note that two Ss showed an increase in $\mathrm{HR}$ in the $4 \mathrm{C}$ condition of the previous investigation. These Ss were the two with the lowest $1 \mathrm{C} \mathrm{HR}$, Ss 5 and 6 . It would appear then that when forms presented singly are barely perceptible and the nature of the forms is such that confusion errors are infrequent, the addition of forms to a display increases HR. Perhaps there is an increase in simultaneous HRs for those Ss who are at the very lowest single form $H R$ because the addition of forms in the display would increase the likelihood of their getting even one look at the form. However, this increase does not approach the increase expected from a model that assumes that each additional form gives the $S$ an additional independent "look." Indeed the 1F4 HRs exceed the 4F HRs indicating that Ss perform better given four successive "looks" than when presented four simultaneous forms. The pattern of results of this study, taken together with the results of the previous study, seems to demonstrate rather conclusively that a $S$ who is presented four stimuli simultaneously does not get four independent looks at the form.

It is clear from the above results that energy level of stimulus presentation and confusion errors play a significant role in determining the HRs for multiple form displays, as well as the complexity of the stimulus forms. This finding adds even more to the difficulty of using HRs in isolation to infer the nature of processing multiple form displays. A simple parallel or serial processing model is obviously inadequate. It can be concluded from this study and that of Keeley and Doherty (1968) that Ss do not process simultaneously presented multiple single-featured forms in parallel. How they do process them is a question that remains as yet unanswered. It seems to the authors that the question is, at the present state of psychological knowledge, not answerable if only HRs are used as a source of inference. There are, as our studies seem to indicate, major methodological difficulties associated with the roles of energy level, confusability, and dimensionality. These are, in principle, solvable problems that could be resolved by a rather massive, multivariate, parametric study. However, there is a theoretical problem to which we alluded in our previous study. The authors believe that the ultimate resolution of the question of parallel processing is dependent upon the prior resolution of the question of how a human $\mathrm{O}$ combines independent pieces of information in arriving at a decision.

\section{REFERENCE}

KEELEY, S. M., \& DOHERTY, M. E. Simultaneous and successive presentations of single-featured and multi-featured visual forms: Implications for the parallel processing hypothesis, Perception \& Psychophysics, 1968, 4, 296-298.

\section{NOTE}

1. Address: Bowling Green State University, Bowling Green, Ohio 43402.

(Accepted for publication January 13, 1969.) 